\title{
EDUCAÇÃO AMBIENTAL E O \\ DESCARTE IRREGULAR DE RESÍDUOS SÓLIDOS URBANOS NA AMAZÔNIA
}

\author{
Carlos José Capela Bispo ${ }^{1}$ \\ Hyago Elias Nascimento Souza ${ }^{2}$ \\ Macella Antônia Pinheiro Monteiro ${ }^{3}$ \\ Júlia Gonzaga Silva da Silva ${ }^{4}$ \\ Kewin Guimarães Machado ${ }^{5}$ \\ Rubens Cardoso da Silva ${ }^{6}$
}

Resumo: Desenvolvido a partir de um Projeto de Extensão juntamente ao Programa de Gestão UEPA Ambiental da Universidade do Estado do Pará com objetivo de atuar no combate ao descarte irregular de resíduos sólidos urbanos em vias públicas da cidade de Belém-PA, utilizando ações práticas de Educação Ambiental. Foi realizado a revitalização das calçadas e vias públicas como ação principal de impacto social. A metodologia aplicada com ações práticas de Educação Ambiental foi eficaz para coibir o descarte irregular de resíduos sólidos urbanos nas vias públicas de Belém gerando um impacto significativo na mudança de hábito da população.

Palavras-chave: Mudança de Hábito; Sensibilização Ambiental; Práticas Ambientais; Vias Públicas.

Abstract: Developed from an Extension Project together with the UEPA Environmental Management Program of the University of the State of Pará with the objective of working to combat the irregular disposal of solid urban waste on public roads in the city of Belém (PA, Brazil), using practical actions of Environmental Education. The revitalization of sidewalks and public roads was carried out as the main social impact action. The methodology applied with practical Environmental Education actions was effective in preventing the irregular disposal of solid urban waste on public roads in Belém, generating a significant impact on the change of habit of the population.

Keywords: Change of Habit; Environmental Awareness; Environmental Practices; Public Roads.

\footnotetext{
1Universidade do Estado do Pará. E-mail: capela@uepa.br. http://lattes.cnpq.br/1082753191579268 2Universidade do Estado do Pará. E-mail: eng.hyagosouza@gmail.com. http://lattes.cnpq.br/9712810427104066 3Universidade do Estado do Pará. E-mail: macella.mapmap@gmail.com. http://lattes.cnpq.br/9837652853977290 4Universidade do Estado do Pará. E-mail: juhh.silvaa483@gmail.com. http://lattes.cnpq.br/9272946372954818 5Universidade do Estado do Pará. E-mail: kewingui.machado@gmail.com. http://lattes.cnpq.br/0614117867039139 6Universidade do Estado do Pará. E-mail: rubens.cardoso@uepa.br. http://lattes.cnpq.br/2298508653005733
} 


\section{Introdução}

A ausência de um gerenciamento eficaz de resíduos sólidos nos centros urbanos e a pouca sensibilização ambiental da população desencadeia uma problemática visível e prejudicial para o meio ambiente: o descarte irregular de Resíduos Sólidos Urbanos (RSU) em vias públicas (DHOKHIKAH et al., 2015, p.153).

Os resultados deste descarte irregular são diversos pontos de resíduos sólidos acumulados em calçadas e vias públicas que desencadeiam problemas socioambientais, de saneamento e de saúde pública, além do impacto visual negativo gerado por este cenário. No Brasil, as estatísticas demonstram uma crescente geração de RSU a cada ano. Essa geração acelerada afeta negativamente a gestão de resíduos sólidos e, propõem novos desafios nos métodos utilizados para sua aplicação (SANTOS, GONÇALVES-DIAS, 2012).

Nesse sentido, a Educação Ambiental (EA) é utilizada como uma ferramenta para potencializar ações educativas que estimulem a conscientização e sensibilização ambiental para minimizar o descarte irregular de RSU (FEITOSA et al., 2019, p.179). Ações práticas melhoram a apropriação dos conhecimentos teóricos sobre sustentabilidade ambiental repassados para a comunidade, tornando essas ações mais significativas e com maior impacto social na população (SAUGO, FERNANDES, 2013: p.65; SANTOS et al., 2016, p.58).

A gestão de resíduos sólidos tem como principal arcabouço legal a Política Nacional de Resíduos Sólidos (PNRS), Lei n. 12.305 de 05 de agosto de 2010. A PNRS destaca a EA como ferramenta fundamental na implantação e eficiência de Planos de Gestão de Resíduos Sólidos (PGRS). A Política Nacional de Educação Ambiental (PNEA), Lei 9.795 de 28 de abril de 1999, define EA como processos por meio dos quais tantos indivíduos quanto a coletividade constroem valores sociais e habilidades voltadas à conservação do meio ambiente e sua sustentabilidade.

Isso reforça como a EA pode ser uma eficaz tecnologia na implantação de PGRS, contribuindo no processo de sensibilização ambiental e na mudança de hábito da população perante a problemática do descarte irregular de RSU (OLIVEIRA et al., 2016, p.285).

Desta forma, mesmo que a EA seja aplicada na gestão de RSU, isso não implica automaticamente que as populações que passaram por processos de conscientização ambiental irão adotar práticas e ações sustentáveis. Embora haja o comprometimento da população em participar da gestão de RSU, seus hábitos socioculturais podem coibir a adoção de práticas sustentáveis, impossibilitando a solução da problemática (DHOKHIKAH et al., 2015, p.154; MARTIN et al., 2006, p.362).

Nesse sentido, a EA deve ser utilizada de forma integrada e contínua, em um processo de conscientização que sensibilize a população na adoção de hábitos sustentáveis em suas atividades diárias (HERMAN et al., 2018). 
Mudanças no "comportamento ecológico" das pessoas irão promover incorporação de escolhas sustentáveis. Primeiro com desafio ecológico, que propõem atuar não apenas em jovens e crianças, mas também em atores sociais que possuam poder para tomadas de decisão pública. Segundo com o desafio social que propõem mudanças radicais na estrutura de uso e gestão dos recursos naturais pela humanidade (VARELA-CANDAMIO et al., 2018, p.1566).

Briggs et al. (2019 p.162) ressalta que a mudança de comportamento é considerada prioridade fundamental nos programas de EA. Entretanto, os estudos e programas de Educação Ambiental geralmente se concentram em repassar conhecimento teórico e não realizam ações práticas contextualizando projetos práticos de extensão como ações que podem trazer impacto significativo no comportamento da população.

Dentro da problemática do descarte de RSU, o Instituto Lixo Zero Brasil (ILZB) realiza ações práticas de EA em parcerias com diversas instituições públicas e privadas. Fundado em 2010, O ILZB representa no Brasil a ZWIA Zero Waste International Alliance, movimento internacional de organizações que desenvolvem o conceito e princípios Lixo Zero no Mundo. Trazendo como missão articular, mobilizar e provocar novas atitudes nas comunidades nacionais e internacionais e promovendo a prática do Lixo Zero nos diversos segmentos da sociedade através da EA (ILZB, 2019).

Essas iniciativas práticas realizadas por Institutos não governamentais e outros grupos apresentam melhores resultados em suas avaliações (HERMAN et al., 2018). No entanto, Carleton-Hug e Hug (2010, p.15) descrevem que boa parte dos programas de EA aplicados pelo mundo tem sido falhos em avaliar os resultados e os impactos na mudança do comportamento social da população. Organizações como a World Wildlife Fund e The Nature Conservancy em projetos voltados a conservação da biodiversidade não têm aplicado avaliação do impacto de suas ações na sociedade (O'NEILL, 2007).

Essa discussão possibilita observar como a EA, aplicada através de ações práticas, pode causar um impacto social mais significativo no comportamento da população. Com isso a percepção ambiental da problemática de RSU é mais bem visualizada e interpretada pela comunidade (OLIVEIRA et al., 2016, p.285).

Desenvolvido a partir de um projeto de extensão juntamente ao Programa de Gestão UEPA Ambiental da Universidade do Estado do Pará (UEPA) com o objetivo de atuar no combate ao descarte irregular de RSU em vias públicas da cidade de Belém-PA, utilizando ações práticas de EA. Além disso, buscou-se trabalhar a revitalização das calçadas e vias públicas como ação principal de impacto social. 


\section{Material e Métodos}

\section{Área de estudo}

O município de Belém está localizado na Amazônia Oriental. É a capital do Estado do Pará na região Norte do Brasil. O município possui aproximadamente uma área de $1.064,918 \mathrm{~km}^{2}$, e está situado entre as coordenadas $01^{\circ} 27^{\prime \prime} 21^{\prime}$ de latitude $S$ e $48^{\circ} 30^{\prime \prime} 16^{\prime}$ de longitude W e altitude de 10 metros (NUNES et al., 2017, p.4788). O bairro do telégrafo foi o alvo do projeto, localizado próximo ao centro da cidade de Belém e onde está situada a Reitoria da Universidade do Estado do Pará (Figura 1).

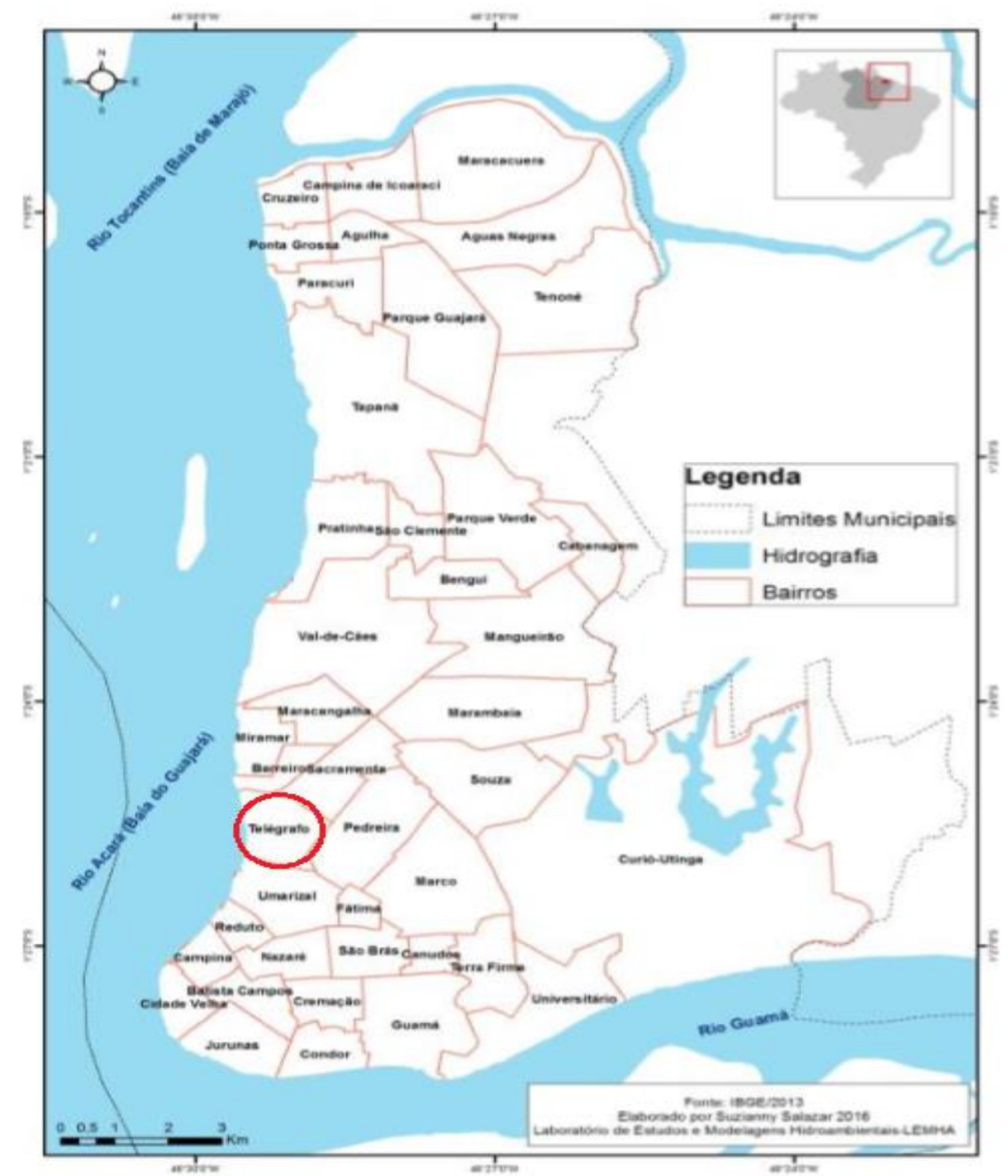

Figura 1: Localização do município de Belém-PA e seus respectivos bairros urbanos.

Fonte: Nunes et al. (2017). 


\section{Metodologia}

A metodologia teve como base a PNRS, Lei n. 12.305/2010 e a PNEA, Lei n. 9.795/1999, sendo dividida em duas etapas.

A primeira etapa consistiu na interpretação da problemática do descarte irregular de RSU na cidade de Belém através do comportamento da população descrito por Varela-Candamio et al. (2018). A partir das análises, foram determinadas atividades de conscientização e sensibilização ambiental através de ações práticas de EA.

Duas escolas públicas foram envolvidas nas atividades a fim de inserir o contexto da educação formal das escolas com os princípios da EA. Uma oficina teórico-prática foi realizada dentro das escolas com o tema "Resíduos Sólidos Urbanos: como cuidar do seu lixo", onde a partir das observações e entendimento dos estudantes, foi aplicado um questionário disponibilizado e adaptado de Santos et al. (2019).

Também foi realizada uma oficina em artes para observar a percepção ambiental dos estudantes através da pintura artística e ensiná-los algumas técnicas básicas de pintura (SANTOS et al., 2015). Essa oficina teve como objetivo principal preparar os estudantes para as atividades práticas e promover uma reflexão interdisciplinar.

A segunda etapa compreendeu no desenvolvimento e aplicação das ações práticas de EA no município de Belém. A primeira ação foi voltada a conscientização e sensibilização dos moradores do bairro do Telégrafo através de visitas na casa dos moradores para o esclarecimento sobre a problemática do descarte irregular de RSU. Também, foi feito convite para participação da ação de revitalização das calçadas e vias públicas próximas as residências. Essas visitas foram essenciais para alertar a comunidade sobre os impactos do descarte de RSU e motivá-los a adotar hábitos para coibir tal descarte (HERMAN et al., 2018).

A ação prática principal teve como objetivo revitalizar as calçadas e vias públicas do bairro do Telégrafo, onde são acumulados irregularmente RSU. Em parceria com a Secretaria Municipal de Saneamento (SESAN) foi realizado um mutirão de limpeza para retirada de todos os RSU; por questões de saúde e segurança essa atividade envolveu apenas Técnicos e Servidores da SESAN.

Posteriormente, foram reaproveitados pneus inservíveis na arquitetura paisagística, doados por empresas automobilísticas. O propósito desta ação foi evitar que o descarte irregular de RSU voltasse a ocorrer nos locais através da criação de uma barreira verde. Essa ação ocorreu em três fases: confecção e pintura artística dos pneus, preparação de substrato com adubo, plantio de mudas ornamentais como descrito por Silva e Fogaça (2017). Pinturas em muros também foram realizadas com intuito de transmitir mensagens de sustentabilidade ambiental através da arte visual (MOURA et al., 2015). 
Essas atividades tiveram duas atuações pontuais. A primeira na Semana Mundial do Meio Ambiente (junho, 2019) e a segunda na Semana Mundial Lixo Zero (outubro, 2019), com a participação de estudantes, servidores públicos, movimentos sociais e da comunidade do entorno.

\section{Resultados e Discussão}

O questionário revelou que os estudantes demonstraram ter conhecimentos sobre o tema RSU. Mais de $70 \%$ dos estudantes responderam que a EA serve para ensinar as pessoas sobre o meio ambiente, o que também foi observado por Santos et al. (2019).

Mais de $50 \%$ dos estudantes não souberam definir o que são RSU, onde a maioria utiliza o termo "lixo". Dentro do conteúdo da palestra foi discutido o termo "resíduos sólidos" como forma mais adequada de definir os resíduos gerados dentro de casa que podem ser reutilizados e reciclados. Quando se trata de crianças e adolescentes a linguagem técnica ambiental deve ser adaptada para que esse público possa absorver melhor o conhecimento repassado no contexto da EA (DEMOLY; SANTOS, 2018).

Todos os alunos informaram que em suas residências não ocorre nenhum tipo de segregação seletiva dos RSU. Este cenário ainda e uma realidade na Região Metropolitana de Belém que não possui coleta seletiva e destina os RSU ao aterro sanitário da região (SOUZA et al., 2019).

Mais de $95 \%$ dos estudantes tem consciência que os RSU podem trazer problemas para a saúde humana e para o meio ambiente e todos responderam que o local de destinar os RSU é "na lixeira" e não em ruas e calçadas.

A análise do questionário deixa claro que os estudantes possuem consciência ambiental e que os mesmos estão dispostos a colaborar no gerenciamento adequado dos seus RSU gerados em casa adotando hábitos sustentáveis. A gestão de resíduos sólidos precisa ser realizada de forma integrada envolvendo desde a administração pública até a participação da população como principal ator no gerenciamento adequado dos RSU (ADENIRAN et al., 2017).

A oficina artística de pintura demonstrou a criatividade e uma visão holística dos estudantes em relação à problemática ambiental. A sensibilização que é transmitida através da arte contribui na formação cultural do ser humano modulando seus hábitos e costumes principalmente referentes às questões ambientais (SANTOS et al., 2015).

A visita nas residências dos moradores do bairro do Telégrafo evidenciou que nenhum deles realiza separação seletiva dos RSU em seus domicílios. Do total dos moradores visitados, $95 \%$ estão conscientes que os RSU podem ocasionar problemas à saúde e ao meio ambiente, entretanto foi observado que $80 \%$ dos moradores descarta irregularmente seus RSU nas 
calçadas. Essa atitude pode ser explicada pela ausência de coleta pública diária de RSU no bairro, visto que apenas há coleta 3 vezes por semana. Diante disso, a população retira seus RSU gerados na residência e descartam nas calçadas.

Combater esse hábito da população é a chave para solucionar esta problemática. Briggs et al. (2019) corrobora que a EA deve adotar ações práticas que proporcione a mudança de comportamento das pessoas. No entanto, Dhokhikah et al. (2015) ressalta a importância de comprometimento e esforço da população em mudar seus hábitos e evitar esse descarte irregular de RSU.

Januario et al., (2017) ao analisar o grau de conscientização ambiental e o comprometimento da população com a coleta seletiva de RSU em Wenceslau Braz-PR, concluiu que quase todos os entrevistados possuem consciência clara sobre conceitos de reciclagem e RSU e que a metade deste público tem interesse neste assunto, no entanto a maioria não adota hábitos sustentáveis ou nenhum comportamento ambiental coerente de pessoas ecologicamente conscientes.

Filha et al., (2018) ao observar a gestão de RSU juntamente com a população do município de Parauapebas-PA, percebeu que existe muito a ser feito em relação a aplicação da gestão eficaz de RSU nos municípios da Amazônia, cabendo aos gestores públicos e a comunidade utilizar de forma efetiva os instrumentos da EA como ferramenta base para minimizar a problemáticas dos RSU.

Nesse sentido, o convite feito a comunidade para participar da ação prática de revitalização das calçadas não foi eficaz, uma vez que apenas $10 \%$ do público convidado compareceu no ato da ação, contudo a participação de crianças e jovens foi significativa representando mais de $80 \%$ do público presente.

O mutirão de retirada e limpeza de RSU das calçadas foi realizado de forma eficaz pela SESAN, deixando o espaço apto para a revitalização. A participação dessa Secretaria foi essencial no sucesso do projeto. A parceria de projetos de extensão, no âmbito dos RSU, com a SESAN é fundamental, pois esse órgão é responsável pelo saneamento ambiental e pelos projetos de EA desenvolvidos pelo município.

Os pneus confeccionados foram distribuídos nos espaços selecionados e posteriormente foi realizada a ação de plantio de espécies ornamentais (Figura 2). Em paralelo, foi realizada a pintura dos muros com imagens que remetessem um impacto visual sobre a problemática e também mensagens sustentáveis (Figura 3). 


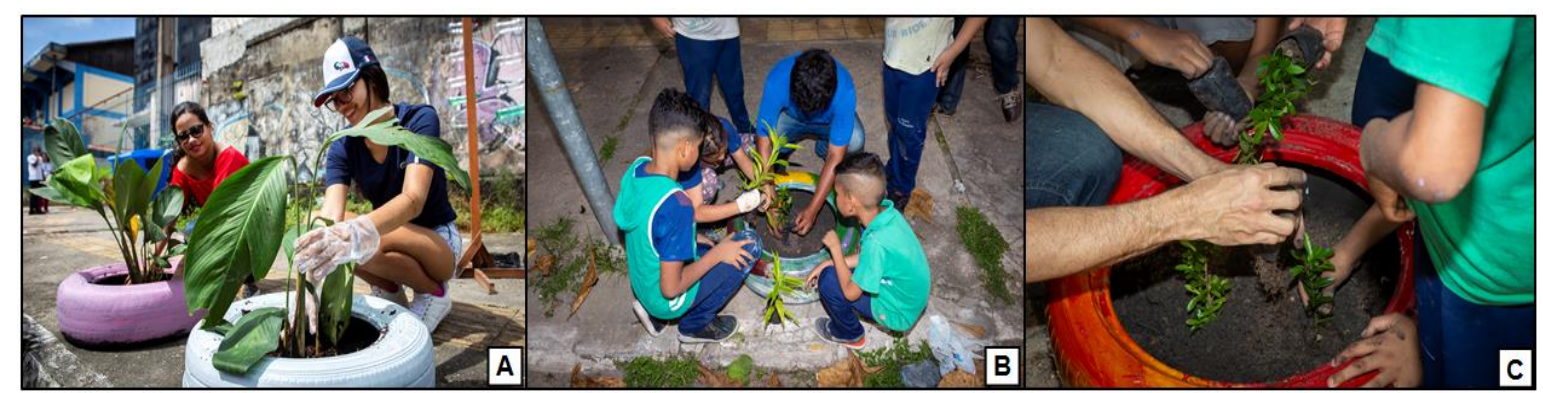

Figura 2: Plantio de mudas realizado pela comunidade $(A)$ e pelos estudantes de escolas públicas (B, C). Fonte: Assessoria de Comunicação da UEPA (2019).

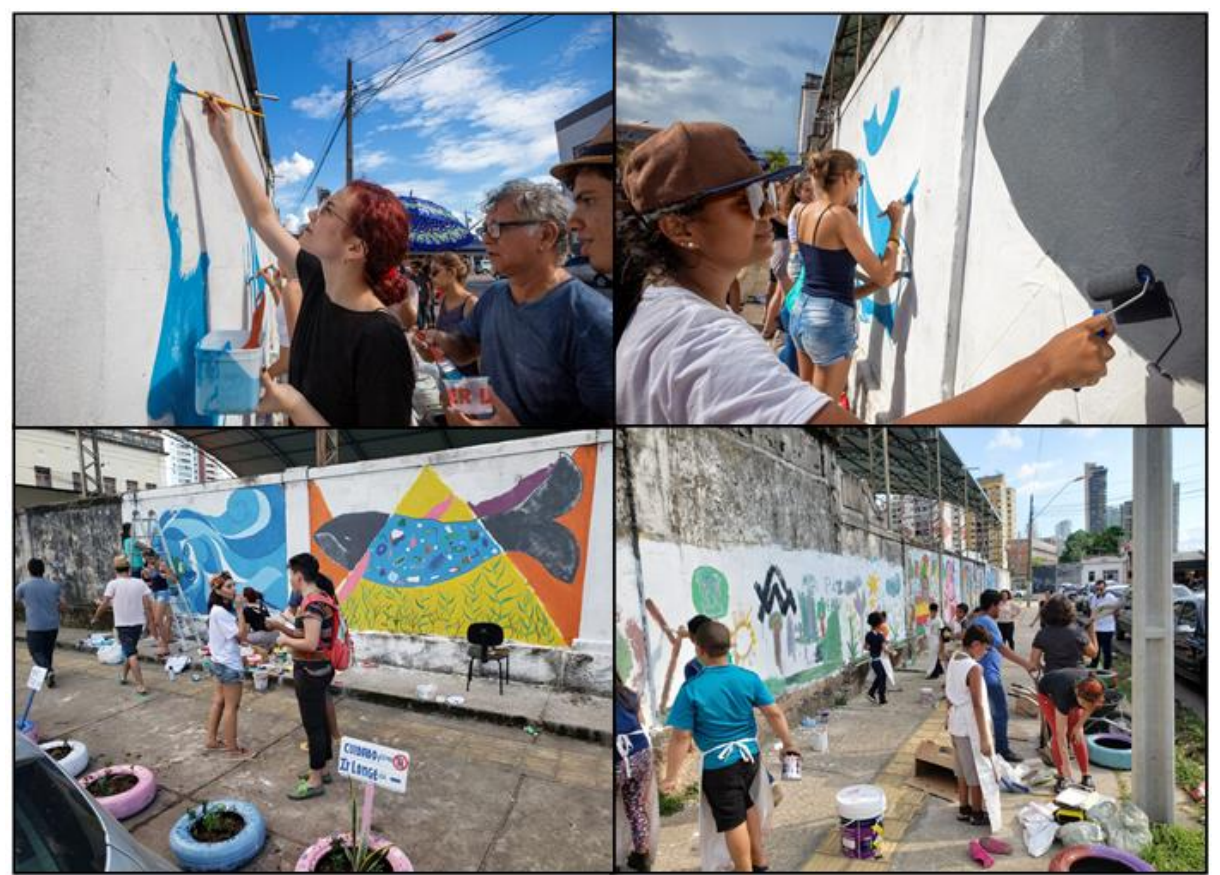

Figura 3: Pintura de muros realizada por estudantes do curso de Design da UEPA e estudantes de escolas públicas. Fonte: Assessoria de Comunicação da UEPA (2019).

Silva e Fogaça (2017) ao desenvolverem a criação sustentável da promoção e a integração de espaços verdes, usando a estética como elemento característico para a transformação da paisagem no âmbito da escola em Manaus-AM, constataram que apesar da preocupação ambiental e da educação presente nas escolas, há uma dicotomia entre a prática e a teoria, visto que nos próprios estabelecimentos de ensino os discentes constatam que não há uma efetiva integração com os espaços construídos.

Com isso, durante a ação buscou-se inserir integralmente os participantes nas atividades práticas. A população que representou a comunidade participou de forma efetiva, colaborando no plantio das mudas e doando mais espécies vegetais que cultivam em suas residências. Vários diálogos foram realizados durante a ação, sempre buscando sensibilizar os participantes a adotar hábitos sustentáveis em sua rotina (Figura 4). 


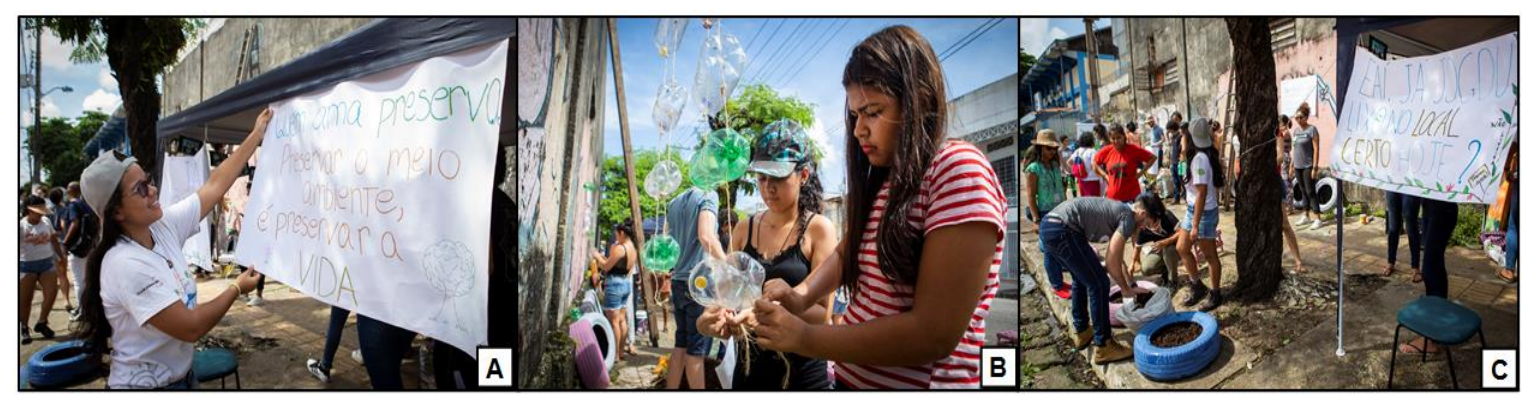

Figura 4: Comunicação visual (A), revitalização utilizando RSU recicláveis (B) e stand para diálogos sustentáveis sobre RSU (C). Fonte: Assessoria de Comunicação da UEPA (2019).

A proposta final da ação foi entregar o espaço revitalizado para que a comunidade que reside próximo cuide, faça a manutenção, fiscalizando para que não haja mais o descarte irregular de RSU, proporcionando bem-estar.

\section{Conclusões}

A metodologia com ações práticas de EA utilizada foi eficaz para coibir o descarte irregular de RSU nas vias públicas de Belém. Após um mês de observação não houve novamente descarte irregular de RSU nos espaços revitalizados. Nesse sentido, conclui-se que houve um impacto significativo na mudança de hábito da população.

Os espaços revitalizados foram bem recebidos pela comunidade, porém não foi observada nenhuma atitude de manutenção pelos moradores locais. A Universidade do Estado do Pará realiza manutenção de limpeza em alguns espaços revitalizados. A Secretaria Municipal de Saneamento realiza manutenções das vias públicas, calçadas e logradouros através de um cronograma interno.

Ficou claro como a EA aplicada de forma teórica e prática em diferentes públicos-alvo se torna mais eficiente na sensibilização ambiental e na mudança de hábitos da população.

\section{Agradecimentos}

Ao Programa de Extensão "Campus Avançado" e ao Programa de Gestão "UEPA Ambiental" da Universidade do Estado do Pará pelo apoio financeiro e logístico. A Escola Estadual de Ensino Fundamental e Médio "Vera Simplício" e Escola Técnica Estadual "Magalhaes Barata" pela participação no projeto. A Secretaria Municipal de Saneamento pelo apoio técnico e a doação de mudas ornamentais. A Secretaria de Estado de Educação (SEDUC), Movimento Jovem pelo Meio Ambiente e ao Grupo de Pesquisa em Educação e Meio Ambiente (GRUPEMA) e ao Instituto Lixo Zero Brasil (ILZB) pelo apoio e participação. 


\section{Referências}

ADENIRAN, A. E.; NUBI, A. T.; ADELOPO, A. O. Solid waste generation and characterization in the University of Lagos for a sustainable waste management. Waste Management, v. 67, p. 3-10, 2017.

BRASIL. Lei Federal n. 9.795, de 27 de abril de 1999. Dispõe sobre a Educação Ambiental, institui a Política Nacional de Educação Ambiental e dá outras providências. Diário oficial da União, Brasília, 1999. Disponível em: <http://www.planalto.gov.br/ccivil 03/leis/L9795.html> Acesso: 1 de dez. 2019.

BRASIL. Lei Federal no 12.305, de 2 de agosto de 2010. Institui a Política Nacional de Resíduos Sólidos; altera a Lei no 9.605, de 12 de fevereiro de 1998; e dá outras providências. Disponível em: $<$ http://www.planalto.gov.br/ccivil 03/ ato20072010/2010/lei/l12305.htm>Acess ado em: 30 de nov. 2019.

BRIGGS, L.; TRAUTMANN, N.; PHILLIPS, T. Exploring challenges and lessons learned in cross-cultural environmental education research. Evaluation and program planning, v. 73, p. 156-162, 2019.

CARLETON-HUG, A.; HUG, J. W. Challenges and opportunities for evaluating environmental education programs. Evaluation and program planning, v. 33, n. 2, p. 159-164, 2010.

SILVA, M.S.; FOGAÇA, T.K. Paisagismo com arte uma solução para o ambiente escolar em Manaus-AM. Meio Ambiente e Sustentabilidade, v. 11, n. 6, 2017.

DEMOLY, K.R.A.; SANTOS, J.S.B. Aprendizagem, Educação Ambiental e escola: modos de agir na experiência de estudantes e professores. Ambiente \& Sociedade, v. 21, p. 1-20, 2018.

DHOKHIKAH, Y., TRIHADININGRUM, Y., \& SUNARYO, S. Community participation in household solid waste reduction in Surabaya, Indonesia. Resources, Conservation and Recycling, v. 102, p. 153-162, 2015.

SANTOS, J.D.; ÁVILA, M.L.D.; SEIDEL, M.I.S.; COSTA, N.; MOURA, S.E.V. Artes plásticas e Educação Ambiental: uma reflexão e sensibilização interdisciplinar. Revista Monografias Ambientais, v.14, n.1, p.123-135, 2015.

FEITOSA, A. K.; BARDEN, J. E.; KONRAD, O. Educação Ambiental: uma experiência com resíduos sólidos domiciliares. Revista Brasileira de Educação Ambiental, v. 12, n. 2, p. 178-183, 30 jun. 2017.

FILHA, M. D. C. V., DE SOUSA, E. A. F., \& DA PAIXÃO, A. J. P. Educação Ambiental e gestão de resíduos sólidos residenciais no município de Parauapebas (PA). Revista Brasileira de Educação Ambiental, v. 13, n. 2, p. 104-120, 2018.

HERMAN, B. C.; SADLER, T. D.; ZEIDLER, D. L.; NEWTON, M. H. A socioscientific issues approach to environmental education. In: International perspectives on the theory and practice of environmental education: $A$ reader (pp. 145-161). Springer, Cham, 2018. 
JANUARIO, M., FERNANDES, F. R. M., VALERIO, M. A., \& MACEDO, R. B. A study of environmental behaviour of the Wenceslau Braz (in the state of Parana) population as regards urban solid waste. Revista de Gestão Ambiental e da Sustentabilidade, v. 6, n. 1, p. 55-72, 2017.

MARTIN, M.; WILLIAMS, I.D; CLARK, M. Influências sociais, culturais e estruturais na reciclagem de resíduos domésticos: um estudo de caso. Recursos, conservação e reciclagem, v. 48, n. 4, p. 357-395, 2006.

MOURA, J.D.P.; MOREIS, C.S.; RODRIGUES, V.H. Grafitando muros escolares, produzindo territórios criativos. Revista Ciência Geográfica, v. 19, p. 153-162, 2015.

NUNES, L. P. M.; SANTOS, J. S.; SILVA, S. C. S.; PIMENTEL, M. A. Variação da temperatura do solo no leste da Amazônia: Os Desafios da Geografia Física na Fronteira do Conhecimento, v. 1, p. 4787-4797, 2017.

OLIVEIRA, B. O. S. DE; GRAÇA, V. R.; SILVA, D. M. P. DA; PAES, L. F. Percepção ambiental de alunos da educação de jovens e adultos sobre o gerenciamento de resíduos sólidos urbanos em Humaitá (AM). Revista Brasileira de Educação Ambiental, v. 11, n. 1, p. 284-304, 2016.

O'NEILL, E. Auditorias de Conservação: Lições Aprendidas no Processo de Auditoria, 2003-2007: Conservations Measures Partnership. 2007. Disponível online em <http://conservationmeasures.org/CMP/Site Docs/Conservation\%20Audits \%20FINAL\%20DRAFT\%2031\%20July\%202007.pdf>.

SANTOS, M.C.L.; GONÇALVES-DIAS, S.L.F.G. Resíduos Sólidos Urbanos e seus impactos socioambientais. São Paulo: IEE-USP, 2012.

SANTOS, S. L. F.; DINIZ, R. G.; SANTOS, G. O. Resíduos eletrônicos: conscientização, campanhas e benefícios socioambientais. Revista Brasileira de Educação Ambiental, v. 14, n. 3, p. 238-251, 2019.

SANTOS, V.S.; SCHMITT, J.L.; ROSA, M.D. A Educação Ambiental como potencial para o gerenciamento de resíduos sólidos escolares: o caso da emef boa saúde, Novo Hamburgo (RS). Revista Brasileira de Educação Ambiental, v.11, n.5, p.53-66, 2016.

SAUGO, A.; FERNANDES, L. C.. Ensino e extensão: relato de uma experiência didática voltada para a sustentabilidade. Revista de Arquitetura da IMED, v. 2, n.1, p. 56-66, 2013.

SOUZA, H.E.N.; BISPO, C.J.C.; SARDINHA, A.S.; NOGUEIRA, C.J.L.G.; COSTA, D.C.T.; SILVA, E.M.; THIJM, F.S.A.; SANTANA, T.M.; ROSA, V.L.G. Gestão de resíduos sólidos em uma Universidade na Amazônia. Anais do 10을 Fórum Internacional de Resíduos Sólidos do Instituto Venturi Para Estudos Ambientais. João Pessoa:Instituto Venturi de Estudos Ambientais. p. 1-9, 2019.

VARELA-CANDAMIO, L.; NOVO-CORTI, I.; GARCÍA-ÁLVAREZ, M. T. The importance of environmental education in the determinants of green behavior: $A$ meta-analysis approach. Journal of Cleaner Production, v. 170, p. 15651578, 2018. 